Best Pract Res Clin Endocrinol Metab. 2014 January ; 28(1): 59-69. doi:10.1016/j.beem.2013.11.005.

\title{
Multifaceted roles of Adiponectin in cancer
}

\author{
Lionel Hebbard, Ph.D. ${ }^{\mathrm{a}, 1}$ and Barbara Ranscht ${ }^{\mathrm{b},{ }^{*}}$ \\ aStorr Liver Unit, Westmead Millennium Institute and The University of Sydney, PO Box 412, \\ Darcy Road, Westmead, NSW 2145, Australia \\ bSanford-Burnham Medical Research Institute, NIH-designated Cancer Center, Tumor \\ Microenvironment Program, 10901 N. Torrey Pines Road, La Jolla, CA 92037, USA
}

\begin{abstract}
Obesity is linked to increased cancer risk. Pathological expansion of adipose tissue impacts adipocyte function and secretion of hormonal factors regulating tissue homeostasis and metabolism. Adiponectin is an adipocytesecreted, circulating hormone with pleiotropic functions in lipid and glucose metabolism, and beneficial roles in cardiovascular functions and inflammation. In obesity, decreased Adiponectin plasma levels correlate with tumor development and progression. The association of Adiponectin with potential tumor-limiting functions has raised significant interest in exploring this adipokine as a target for cancer-diagnostic and therapeutic applications. Recent studies, however, also implicate Adiponectin in supporting malignancy. This review highlights the evidence that links Adiponectin signaling to either cancer-protective or cancer-supporting functions. In this context, we discuss Adiponectin interactions with its receptors and associated signaling pathways. Despite significant advances in understanding Adiponectin functions and signaling mechanisms, its role in cancer remains multifaceted and subject to controversy.
\end{abstract}

\section{Keywords}

Adiponectin; AdipoR1; AdipoR2; T-cadherin; cancer; signaling

\section{Introduction}

Adipose tissue plays an important role in regulating energy metabolism and maintaining tissue homeostasis. Distributed in the visceral cavity, subcutaneously, and around many organs, adipocytes store excess energy in form of lipid, and regulate lipolysis upon energy demand. Adipose tissue is also an active endocrine organ that secretes a range of bioactive factors to exert endocrine and paracrine functions in diverse tissues. These factors referred to as adipokines, balance numerous physiological processes, including glucose- and lipid metabolism, insulin signaling, and inflammation. Obesity represents a pathological state

\footnotetext{
"Corresponding author. Tel.: +1 858646 3100, +1 8586463122 (direct line); Fax: +1 8586463197.

$1_{\text {Tel.: }+61298459132 .}$

Conflicts of interest

The authors declare no conflicts of interest.
} 
during which adipose tissue undergoes massive expansion primarily through adipocyte hypertrophy that restricts vascularization and leads to hypoxia, invasion of inflammatory cells and fibrosis [1]. Pathological fat accumulation is associated with oxidative- and endoplasmic reticulum stress and malfunctions of the unfolded protein response that impair the principle adipocyte functions in regulating of lipid storage and adipokine secretion [2].

Metabolic Syndrome (MetS) characterized by combinations of central obesity, dysregulated lipid- and glucose metabolism, insulin resistance, Type-2 diabetes, hypertension, cardiovascular disease, or chronic tissue inflammation, is linked to adipocyte dysfunctions [3]. Adiposity and MetS present an increased risk for obesity-related cancer and cancerassociated mortality $[4,5]$.

One of the major links between obesity and cancer is aberrant secretion and signaling of adipokines. Adiponectin (APN) is a major fat-secreted, circulating adipokine with pleiotropic, beneficial functions in human health [6-8]. APN positively balances glucose and lipid metabolism, and has insulin-sensitizing, anti-apoptotic and anti-inflammatory properties [9]. APN circulating levels decline in obesity and MetS, while insulin-, leptin-, growth factor- and pro-inflammatory adipokine secretion and associated signaling increase. Several lines of evidence suggest that increasing circulating APN levels, or activating APNinduced signaling pathways can counteract metabolic dysfunctions and slow cancer progression in experimental models [10-12]. Thus, restoring the balance of adipokine production, and particularly mimicking the cancer-protective effects of APN observed in these studies, has attracted significant interest for potential clinical applications. However, research on APN has provided evidence for both positive and negative influences of APN on tumor growth. Thus, understanding APN's complex biology and diverse signaling mechanisms, and linking its role to cancer present challenging tasks. This article summarizes the status of APN research in cancer, and aims to complement several excellent recent reviews on this topic [13-15]. For space restrictions, citations are limited to only representative examples illustrating specific points.

\section{Adiponectin: Biology and Receptors}

\section{Adiponectin}

Adiponectin is a multimeric protein that exists in different, biologically active isoforms. Basic $30 \mathrm{kD}$ monomeric subunits consist of an $\mathrm{N}$-terminal collagenous domain and a globular head structurally related to complement factor C1q/Tumor Necrosis Factor (TNF)family proteins [16-18]. Pre-secretion, posttranslational processing generates trimers, trimer-dimers (hexamers), and high molecular weight (HMW) APN. HMW-APN, the dominant form in plasma, assembles from multiple trimers into higher order structures resembling a "bouquet" with a collagenous stalk and globular "blossoms" [19-23]. HMWAPN is considered physiologically most relevant, and increasingly serves as a marker for disease-associated adipocyte dysfunctions [23,24]. In lean, healthy individuals, circulating APN concentrations are high compared to other hormones and growth factors, with 3-30 $\mu \mathrm{g} / \mathrm{ml}$, or $0.01 \%$ of the total serum protein [18]. Levels are regulated by numerous factors, including genetics, nutritional-, and inflammatory status, posttranslational modifications, 
binding to receptors and mechanisms affecting clearance [25-27]. Influenced by hormones, women generally show higher total and HMW-APN than men [9].

Different APN isoforms have been utilized for experimental purposes. The source of the recombinant APN protein (eukaryotic or prokaryotic), the quality of the preparation, and the degree of posttranslational processing and multimerization significantly affect experimental outcome [28]. Generation of higher order APN multimers requires proper post-translational processing by eukaryotic cells [19-22,29]. Numerous in vitro studies have utilized bacterially-produced recombinant protein representing the APN globular domain (gAPN). However, since gAPN is not detected endogenously, its roles are insufficiently connected to APN's in vivo physiology. Local cleavage and short-lifespan of gAPN have been proposed, but not proven, to reconcile this discrepancy [30,31].

\section{Adiponectin Receptors}

Plasma levels of APN in lean healthy persons are three orders of magnitudes higher than the level of growth factors and cytokines that interact with conventional receptors. Thus, APNreceptor interactions must be different from classical, high affinity receptor-ligand interactions. A decade ago, two unconventional heptahelical APN receptors, Adiponectin Receptor 1 (AdipoR1), and Adiponectin Receptor 2 (AdipoR2) with the inverted membrane topology of G-protein-coupled receptors were identified by expression cloning [32]. Moreover, a novel task in APN binding was discovered for glycosylphosphatidyl inositol (GPI)-anchored T-cadherin, a cell adhesion protein that was known for its calciumdependent, homotypic binding functions that are typical for the classical cadherins [22,33$35]$.

AdipoR1 and AdipoR2-The seven-span AdipoR1/R2 bind different APN isoforms with different affinities. AdipoR1 is a high-affinity receptor for gAPN and a low affinity receptor for full-length APN, while AdipoR2 binds both isoforms with intermediate affinity [32,36]. AdipoR1/R2 belong to the functional category of progesterone and Adiponectin Q receptors (PAQR), that like some members of this family, possess ceramidase activity [37].

Physiologically, engagement of APN with AdipoR1/2 increases phosphorylation of AMPactivated protein kinase (AMPK), and suppresses energy-consuming processes such as lipogenesis and gluconeogenesis [32,36]. A second pathway, mainly activated by APN interactions with AdipoR2, is regulation of peroxisome proliferators-activated receptor a (PPARa) ligand activity to increase fatty acid combustion [32,36]. Both of these pathways are activated under conditions of energy stress, and increase catabolic processes to renew cellular energy.

T-cadherin/CDH13-T-cadherin, encoded by the human CDH13 gene, was recognized as an APN-binding protein by its ability to bind hexameric and HMW-APN isoforms [22]. Tcadherin was discovered in our laboratory as a unique "truncated" cadherin that is associated with the plasma membrane through a glycosyolphosphatidyl-inositol (GPI) moiety [33,34]. Since T-cadherin lacks cytoplasmic sequences, it falls short of a classical receptor with both ligand-binding and intracellular signaling capabilities. Rather, T-cadherin's main role in vivo is docking APN to responsive tissues, as shown for heart, muscle and vasculature. In T- 
cadherin-deficient (Tcad-KO) mice, physiologically composed APN accumulates at more than 4-fold its normal level in the circulation [38,39]. Heart and vascular tissues lacking Tcadherin become insensitive to APN despite continued expression of AdipoR1/R2 [39,40]. Similar to APN-deficient mice, Tcad-KO mice show ischemia-induced heart and vascular dysfunctions and develop metabolic dysregulation [39-41].

Genetic studies seeking to identify alterations in genes associated with low APN levels, identified strongest statistically significant single nucleotide polymorphisms (SNP) in CDH13, the gene encoding human T-cadherin [42,43]. Consistent with this implicated link, $\mathrm{T}$-cadherin protein expression is down-regulated from heart, muscle and endothelial cells in mice lacking APN [39,40]. Treatment of APN-KO mice with recombinant APN (rAPN) restores T-cadherin expression on cell surfaces to normal levels, and this is prerequisite for the "rescue" of cardiac and angiogenic phenotypes in APN-KO mice [39,40]. The signaling pathways activated through T-cadherin downstream of APN include activation of AMPK $[39,40]$ through yet unknown mechanisms.

\section{Roles of Adiponectin in cancer}

Genomic instability and environmental factors are recognized as major mechanisms contributing to cancer etiology [44]. Genetic lesions enact cellular programs in neoplastic cells to evade immune surveillance, survive and meet increased energy demand for growth and proliferation. Tumor cells foster constant cross-talk with their environment to coerce nutrients and oxygen for self-propagation, and adjust metabolic functions and signaling mechanisms to self-renew, migrate, metastasize and resist apoptosis [44,45]. Circulating hormones and growth factors can also directly regulate tumor cell growth, or influence tumorigenesis through modifications of the stromal microenvironment composed of fibroblasts, immune- vascular and other cells. APN has attracted attention in the cancer field as a large number of studies correlate increased cancer risk, progression and mortality with low circulating levels of this adipokine [13]. Studies in cancer cell lines reiterated growthinhibitory roles of APN, and contributed associated signal transduction pathways. However, newer studies increasingly challenge the concept of APN's tumor suppressive functions. How are these contradicting roles regulated? We discuss in this context major aspects of APN functions and cellular signaling mechanisms.

\section{Circulating Adiponectin levels in cancer patients}

Clinical studies have established the correlation between low APN levels and obesity-linked cancers [13]. Primarily affected are cancers of the digestive system (esophageal adenocarcinoma, gastric, liver and colorectal cancers), hematological cancers (leukemia, lymphoma and myeloma) and hormonally influenced cancers (post-menopausal breast cancers, endometrial and prostate cancers) [13,14]. The data correlate low serum APN with increased numbers and size of tumor foci [46], and elevated invasion and malignancy stage [47]. In lung cancer patients, circulating APN concentrations were indistinguishable from healthy controls at early stages, but declined in patients with advanced disease [48]. A shift in APN isoforms to prominently trimeric (low molecular weight, LMW) APN was noted in Japanese patients and linked with decreased hepatocellular carcinoma (HCC) [49]. 
While the preponderance of evidence suggests an inverse relation of APN to malignancy, another group of studies correlates increased APN levels with cancer progression. In patients with viral infections or chronic inflammation, increased levels of APN are predictive of cancer development. A greater incidence of liver cancer in patients with chronic hepatitis $\mathrm{C}$, for example, correlates with increased total APN composed of a higher than normal proportion of LMW APN [50]. High APN levels were also noted in pancreatic cancer patients [51] although no uniform results were obtained (reviewed in [52]). High APN levels are linked to all-cause mortality, and may represent a general marker for body wasting, or be compensatory for inflammatory conditions independent of obesity and MetS [53].

Available epidemiological studies raise the question whether APN levels can reliably indicate specific malignancies. Indeed, low APN plasma levels associate with cancers linked to obesity and sex steroid hormones, while enhanced APN serum concentrations seem to indicate inflammatory status and advanced stages of malignancy [13,52]. Alternatively, APN's pleiotropic functions may include only a limited or specific role in cancer, with plasma levels reflecting overall metabolic and inflammatory status of the body. Systemic APN levels thus could serve as a variable for these latter conditions and inform personalized cancer treatments. Linking APN levels and malignancy will require further studies.

\section{Expression of Adiponectin Receptors in tumors}

Altered APN levels in cancer pose the question as to receptor expression and APN binding in cancer tissues. AdipoR1/R2 are widely expressed in most cancers although levels may vary $[13,48,51,54,55]$. In contrast, the T-cadherin-encoding CDH13 gene is silenced in many cancers through hypermethylation [56]. Loss of T-cadherin is linked to increased tumorigenicity and cancer progression in vivo. Reflecting the in vivo expression pattern in tumors, most cancer cell lines commonly utilized for experimental studies express AdipoR1/R2, but not T-cadherin [56]. The introduction of T-cadherin into cancer cells decreases the malignant properties in breast, prostate, and melanoma cancer and hepatocellular carcinoma cell lines in vitro and in tumor xenograft models [56]. Thus, reexpression of T-cadherin in tumor cells can repress cancer-promoting pathways [56]. Analyses of APN dysfunctions in cancer so far have left the role of T-cadherin as a potent APN-binding and anti-tumorigenic protein unconsidered. Therefore, future clinical and epidemiological studies of APN in cancer must take into account the expression of AdipoR1/R2 and T-cadherin in cancers related to APN dysfunctions.

\section{Adiponectin-activated mechanisms limiting tumorigenesis}

Roles of APN in cancer cannot be seen in isolation but must be placed into the context of disrupted metabolic homeostasis resulting from adipocyte dysfunctions, activation of epigenetic pathways in neoplastic cells, and the tumor microenvironment. Tumorigenesis and progression under conditions of low APN levels are linked to the disruption of APNsignaling through AMPK that inhibits proliferative pathways in normal cells. APN also has strong insulin-sensitizing and balancing actions, and decreases in plasma increase insulin signaling associated with neoplasia [7]. Leptin, a metabolic adipokine opposing APN functions is increased under conditions of dysregulated APN and insulin, and associated with tumor progression. Further, declines in APN are commensurate with increases in 
proinflammatory adipokines, including tumor necrosis factor alpha (TNFa and interleukin-6 (IL-6)) that can promote malignancy $[4,5,10,11,57,58]$. In the next sections, we discuss major signaling pathways associated with APN in cancer.

AMPK activation by Adiponectin restricts tumor growth-Insights into the signaling pathways of APN in cancer were gained from studies with cancer cell lines and rodent xenograft models. A major pathway associated with APN's protective functions is activation of AMP-activated protein kinase (AMPK), a central sensor and regulator of cellular energy. Under metabolically challenging conditions such as glucose deprivation, hypoxia and oxidative stress, activation of AMPK shuts down proliferative and growthsupporting pathways, while turning on catabolic pathways to increase ATP (adenosine triphosphate) production [59]. AMPK is considered cancer-protective as activation potently inhibits cell cycle progression and growth [60] (Fig. 1).

In many cells, including cancer cells, APN activates AMPK through interactions with AdipoR1/R2 [61,62]. Receptor activation translocates the Ser/Thr liver kinase B1 (LKB1) from the nucleus to the cytoplasm where in association with the adaptor protein APPL1, LKB1 directly phosphorylates AMPK [61,62]. LKB1 is a tumor suppressor that is regularly mutated in human epithelial cancers and is located upstream of mammalian target of rapamycin (mTOR) pathway [63,64] (Fig. 1).

Downstream, AMPK intersects with diverse signaling pathways. AMPK phosphorylation on Thr172 inhibits acetyl-CoA carboxylase (ACC1/ACC2) to increase import and $\beta$-oxidation of free fatty acids in mitochondria [65]. In addition, APN-AMPK signaling inhibits lipid biogenesis through down-regulation of fatty acid synthase (FAS) $[65,66]$. FAS is upregulated in malignant breast cancer, and the inhibition of either ACC1 or FAS activates AMPK and suppresses tumor cell growth in rodent xenograft models [67,68]. Consistently, RNA interference studies document that inhibition of ACC1 or FAS activities restrict growth and survival of tumor cell lines in vitro [69-71]. APN-mediated AMPK activation can thus inhibit tumor growth and survival dependent on lipid biosynthetic pathways.

However caution is warranted, as APN-mediated activation of the LKB1-AMPK pathway can increase glucose transporter GLUT4 expression and ultimately glucose uptake [61,62]. Increased glucose uptake and utilization are prominent hallmarks of cancer cells, and enhanced glucose transporter activity is associated with cancer progression, particularly at early stages of tumorigenesis [72]. Consequently, under yet poorly understood conditions, activation of AMPK through APN may foster neoplastic cell survival and increase cancer.

Adiponectin balances insulin and growth factor signaling in cancer-It is well documented that insulin supports cancer cell growth and proliferation [73]. Decreased plasma APN is associated with insulin resistance and a marked increase in circulating insulin levels [6-8]. The insulin receptor protein kinase signals through phosphoinositide 3kinase (PI3K) and protein kinase B (AKT) to activate the mammalian target of rapamycin (mTOR) pathway [74]. Aberrant PI3K/AKT/mTOR signaling is widespread in tumorigenesis. Mutations in PTEN (tumor suppressor phosphatases and tensin homolog), 
PI3K, and activation of growth factor receptors, including human epidermal growth factor receptors EGFR and HER2, and fibroblast growth factor receptors affect this pathway.

Importantly, APN is a potent inhibitor of $\mathrm{PI} 3 \mathrm{~K} / \mathrm{AKT} / \mathrm{mTOR}$ that is able to reduce cancer cell growth exerted by insulin and growth factor-induced signaling [75,76] (Fig. 1). APNactivated AMPK intersects with the pro-oncogenic mTOR pathway to suppress cancer growth [77] (Fig. 1). One mechanism is that APN-activated AMPK directly phosphorylates raptor, a constituent of the nutrient- and rapamycin-sensitive mTOR complex 1 (mTORC1), thereby inhibiting mTOR activity. Downstream, mTOR regulates transcription of cancerpromoting hypoxia-inducible factor 1a (HIF1a) cyclin-D1 and c-myc to control cell cycle progression and proliferation. An additional mode of APN to interfere with mTOR signaling is regulating the activity of the tumor suppressor tuberosis sclerosis complex 2 (tuberin). TSC2 reduces cell growth by inhibiting mTOR as the GTPase-activating protein for RHEB (Ras homolog enriched in brain). Loss of TSC2 supports tumors with enlarged cells [78]. Inhibitory phosphorylation of TSC2 and RHEB through growth factor activation of AKT and ERK1/2 (mitogen-activated kinase (MAPK)-family extracellular signal related kinase 1/2) leads to increased mTORC1 activity and promotes tumorigenesis [79]. APN-induced AMPK signaling can counteract these processes.

Finally, APN signaling through AMPK counteracts cell cycle progression associated with growth factor signaling by regulating the activities of tumor suppressor p53, a major gene mutated in cancer. Under conditions of cellular stress, such as glucose limitation, APNAMPK phosphorylates p53 to initiate cell cycle arrest, senescence and apoptosis in cancer cell lines [80]. In colorectal cancer, APN-AdipoR1/R2 increases cyclin dependent kinase inhibitors p21/p27 to induce cell cycle arrest [81,82]. APN can thus suppress cancer cell proliferation through activation of multiple cancer protective genes.

Adiponectin counteracts Wnt signaling in cancer-APN also regulates components of the Wnt signaling pathway to counteract tumorigenesis. Activation of the Wnt growth factor receptor complex promotes colorectal cancer by deregulation of the adenomatous polyposis coli (APC) tumor suppressor complex through glycogensynthase kinase-3 $\beta$ (GSK-3 $\beta$ ) activity [83]. Disruption of the APC- complex stabilizes and subsequently translocates $\beta$-catenin to the nucleus where it induces, as a co-transcriptional activator, cancer promoting cyclin D1, c-myc and other genes [83]. In normal cells, GSK-3 $\beta$ phosphorylates and targets $\beta$-catenin for ubiquitin-induced proteolysis. In cancer cells, disruption of the APC complex through Wnt inactivates GSK-3 $\beta$ thereby promoting tumorigenesis, and regulation of GSK-3 $\beta$ activity through APN halts this process [84]. Treatment of highly invasive, estrogen-receptor (ER)- negative MDA-MB-231 breast cancer cells with eukaryotically-produced full length APN restores GSK-3 $\beta$ phosphorylation and $\beta$ catenin degradation, down-regulates cyclin D1, and reduces the growth of MDA-MB-231 breast cancer cells in a xenograft model [85]. Further studies showed that APN also positively impacts breast cancer downstream of Wnt by upregulating Wnt inhibitory factor-1 to restore $\beta$-catenin homeostasis [86].

\section{Adiponectin antagonizes leptin-induced cancer promoting signals-Under}

conditions of hypoadiponectinemia and hyperinsulinemia, leptin levels coordinately increase 
in the circulation [10]. Leptin supports breast-, pancreatic and gastrointestinal cancer cell proliferation, migration and invasion through receptors on cancer and stromal cells [87,88]. Intriguingly, in a mouse hepatocarcinoma xenograft model, administration of APN decreases leptin-induced cancer progression and angiogenesis by reducing leptin-induced AKT and STAT3 (signal transducer and activator of transcription 3) signaling [89,90]. Upstream, APN upregulates suppressor of cytokine signaling (SOCS2) to inhibit leptin signaling by preventing JAK2 (Janus kinase 2) and subsequent STAT3 phosphorylation of AKT [89]. APN also counterbalances leptin-induced mitogen-activated kinase (MAPK), and estrogen receptor-activated pathways in cancer $[10,89]$, and inactivates leptin-induced Wnt signals [91].

Taken together, these studies highlight evidence that APN counterbalances multiple pathways activated in cancer. APN acts in this capacity directly via AMPK signaling, but also indirectly via its role in maintaining metabolic homeostasis that keeps in check potent growth cytokines, such as Insulin and Leptin, or inflammatory factors, such as TNFa and IL6, that can promote cancer.

\section{Adiponectin-mediated mechanisms supporting tumor progression}

\section{Ceramidase activity and Sphingosine-1 signaling}

While high APN levels are mainly thought to antagonize pathways that promote cancer formation, recent studies indicate that APN could also promote tumor progression. A recently discovered novel mechanism suggests that APN increases ceramidase activity associated with AdipoR1/R2 independent of AMPK [37]. Ceramidase cleaves the fatty acid acyl chain from ceramides and releases sphingosine for phosphorylation to spingosine-1phosphate (S1P). Ceramide and sphingosine are bioactive lipids that are associated with the induction of growth inhibitory pathways, apoptosis, differentiation and cellular senescence [92]. In contrast, S1P promotes cell proliferation and survival, angiogenesis, and cell migration [92]. The relative amounts of ceramide and S1P thus function as a rheostat [93]. S1P secreted from tumor cells binds to S1P receptors on cancer cells to induce growth, survival, invasion and metastasis in an autocrine manner, or acts on cells in the tumor microenvironment to influence cancer progression through paracrine mechanisms $[92,93]$. Extending on this model, expression of high AdipoR1/R2 levels in human cancers would predict tumor-promoting functions for APN. The current data thus implicates AMPK activation through the APN-AdipoR axis in cancer-protective functions, while generation of S1P is linked to cancer promoting pathways. Analyses of AdipoR-deficient mouse genetic models of cancer would help solve this dichotomy.

\section{Adiponectin promotes tumor angiogenesis}

One of the cancer hallmarks is the recruitment of blood vessels to supply the tumor with nutrients and oxygen [44]. Studies by us, and others, have shown prominent pro-angiogenic functions of APN in murine models of breast cancer [94,95]. APN deficient mouse mammary tumor virus-polyoma middle T-antigen (MMTV-PyV-mT) tumors are less vascularized and display increased hypoxia and apoptosis than wildtype tumors. Initially, tumors in APN-KO mice remain smaller than those in their wildtype counterparts. Over 
time, however, the tumors adapt to the increasingly hypoxic environment, assume a highly metastatic phenotype and spread to the lungs. This phenotype mimics pre-clinical results that have shown tumor resistance to anti-angiogenic therapy, and implicated activation of compensatory transcriptional pathways to support angiogenesis and cancer survival [96].

APN mediates its pro-angiogenic functions, at least in part, through T-cadherin [38,39]. Tcadherin associates APN to the vasculature and is necessary for angiogenesis in MMTVPyV-mT tumor- and ischemic hindlimb models where APN-deficient mice show similar phenotypes [39,94,97]. Reversal of the angiogenic phenotype in APN-KO mice through adenovirus-mediated supply of rAPN depends on the presence of T-cadherin [39]. In vitro, both T-cadherin and AdipoR1/R2 are necessary to promote endothelial cell migration and proliferation in response to physiological forms of APN as documented by RNA silencing studies [39]. Studies on APN-mediated signals in endothelial cells implicate AdipoR1/R2, activation of AMPK [97], AKT and endothelial nitric oxide synthase (eNOS) [98], and the RAS-ERK1/2 (extracellular signal-related kinase) mitogen-activated protein kinase (MAPK) pathway [98-100]. Whether T-cadherin and AdipoR1/R2 are functionally linked in regulating angiogenic responses in cancer remains to be determined.

\section{Targeting Adiponectin in cancer}

Since vast literature associates cancer protective functions with APN, biologics to mimic, or drugs to bypass APN by directly activating AMPK are being explored for potential cancer therapies [101]. Trials utilising AICAR show promise to inhibit tumor growth in experimental models of glioblastoma [102]. The recent development of an APN-mimetic peptide agonist that docks into the AdipoR binding pocket showed potency in activating AMPK and decreasing tumorigenesis in a mouse xenograft breast cancer model [103]. Further studies assessing cellular changes and pathology in mouse genetic models of cancer over longer time periods will need to determine utility for clinical trials. Given the recent data, we cannot discern the specific conditions under which APN exerts long-term cancersuppressive functions, or aids cancer cells to adapt to and survive the challenges of a hostile microenvironment. Thus, it is even more important now to rigorously test new drugs targeting APN for cancer protective and promoting functions.

\section{Conclusion}

Pathological regulation of adipocyte-secreted factors and associated changes in metabolism are critical factors in cancer epidemiology. APN is a major circulating adipokine with multifaceted and potentially contradicting functions in tumorigenesis. The complex and multiple roles of APN encompass metabolic regulation, alterations of the tumor microenvironment and direct effects on cancer cells with each of these interactions influenced by additional factors. Functions in limiting tumor growth are underscored by epidemiological studies relating decreases in circulating APN levels to increased malignancy. Moreover, studies with cancer cell lines in vitro and in mouse xenograft models document APN's effects on limiting cell growth and proliferation through specific signal transduction pathways. In contrast, evaluation of APN-deficient mouse genetic models and the identification of associated ceramidase activity with AdipoR1/R2 link APN to increased 
malignancy. Towards understanding these conflicting roles, it is critical to dissect and weigh the cellular and molecular mechanisms by which APN positively and/or negatively affects tumorigenesis in suitable in vivo models.

\section{Open questions}

The APN field is faced with the task of understanding the cellular and molecular basis underlying the controversial functions of APN in cancer. The contributions of endocrine and paracrine functions of APN, and its impact on specific stromal components affecting cancer will need clarification. Alterations not only in circulating levels of total and isoform-specific APN, but also in local, tumor-specific APN concentrations, and the binding to receptors and associated signal transduction pathways on tumor and stromal cells in vivo remain to be explored. In particular, the conditions under which APN through actions on AdipoRs suppresses or activates tumor progression need to be determined. The role of T-cadherin in relation to APN in cancer is a new emerging aspect in the field requiring consideration. In this context, the question of a possible relationship between decreased APN levels and Tcadherin silencing in cancer cells is intriguing. The field will require coherent experimental models to probe short and long-term functions of APN in vivo and mimic specific cellular interactions in suited culture systems.

\section{Acknowledgments}

The authors thank Drs. Christopher Kintner, Stephanie Obeid, and Rainer Stiemer for comments and discussion, and Alexandra Kintner for preparing the Figure. This work was supported by the National Institute of Health, National Cancer Center Grant P30 CA030199 (BR), the National Health and Medical Research Council of Australia Project Grant ID1006200 (LH), Cancer Council of New South Wales IG11-27 (LH), and the Robert W. Storr Bequest to the University of Sydney (LH).

\section{REFERENCES}

1. Sun K, Kusminski CM, Scherer PE. Adipose tissue remodeling and obesity. J Clin Invest. 2011; 121(6):2094-2101. [PubMed: 21633177]

2. Trayhurn P. Hypoxia and adipose tissue function and dysfunction in obesity. Physiol Rev. 2013; 93(1):1-21. [PubMed: 23303904]

3. Guilherme A, Virbasius JV, Puri V, et al. Adipocyte dysfunctions linking obesity to insulin resistance and type 2 diabetes. Nat Rev Mol Cell Biol. 2008; 9(5):367-377. [PubMed: 18401346]

4. Park J, Euhus DM, Scherer PE. Paracrine and endocrine effects of adipose tissue on cancer development and progression. Endocr Rev. 2011; 32(4):550-570. [PubMed: 21642230]

5. Parekh N, Chandran U, Bandera EV. Obesity in cancer survival. Annu Rev Nutr. 2012; 32:311-342. [PubMed: 22540252]

6. Berg AH, Combs TP, Scherer PE. ACRP30/adiponectin: an adipokine regulating glucose and lipid metabolism. Trends Endocrinol Metab. 2002; 13(2):84-89. [PubMed: 11854024]

7. Kadowaki T, Yamauchi T, Kubota N, et al. Adiponectin and adiponectin receptors in insulin resistance, diabetes, and the metabolic syndrome. J Clin Invest. 2006; 116(7):1784-1792. [PubMed: 16823476]

8. Turer AT, Scherer PE. Adiponectin: mechanistic insights and clinical implications. Diabetologia. 2012; 55(9):2319-2326. [PubMed: 22688349]

9. Ye R, Scherer PE. Adiponectin, driver or passenger on the road to insulin sensitivity? Mol Metab. 2013; 2(3):133-141. [PubMed: 24049728]

10. Jarde T, Perrier S, Vasson MP, et al. Molecular mechanisms of leptin and adiponectin in breast cancer. Eur J Cancer. 2011; 47(1):33-43. [PubMed: 20889333] 
11. Vansaun MN. Molecular pathways: adiponectin and leptin signaling in cancer. Clin Cancer Res. 2013; 19(8):1926-1932. [PubMed: 23355630]

12. Grossmann ME, Cleary MP. The balance between leptin and adiponectin in the control of carcinogenesis - focus on mammary tumorigenesis. Biochimie. 2012; 94(10):2164-2171. [PubMed: 22728769]

13. Dalamaga M, Diakopoulos KN, Mantzoros CS. The role of adiponectin in cancer: a review of current evidence. Endocr Rev. 2012; 33(4):547-594. [PubMed: 22547160]

14. Hefetz-Sela S, Scherer PE. Adipocytes: impact on tumor growth and potential sites for therapeutic intervention. Pharmacol Ther. 2013; 138(2):197-210. [PubMed: 23353703]

15. Heiker JT, Kosel D, Beck-Sickinger AG. Molecular mechanisms of signal transduction via adiponectin and adiponectin receptors. Biol Chem. 2010; 391(9):1005-1018. [PubMed: 20536390]

16. Hu E, Liang P, Spiegelman BM. AdipoQ is a novel adipose-specific gene dysregulated in obesity. J Biol Chem. 1996; 271(18):10697-10703. [PubMed: 8631877]

17. Maeda K, Okubo K, Shimomura I, et al. cDNA cloning and expression of a novel adipose specific collagen-like factor, apM1 (AdiPose Most abundant Gene transcript 1). Biochem Biophys Res Commun. 1996; 221(2):286-289. [PubMed: 8619847]

18. Scherer PE, Williams S, Fogliano M, et al. A novel serum protein similar to C1q, produced exclusively in adipocytes. J Biol Chem. 1995; 270(45):26746-26749. [PubMed: 7592907]

19. Shapiro L, Scherer PE. The crystal structure of a complement-1q family protein suggests an evolutionary link to tumor necrosis factor. Curr Biol. 1998; 8(6):335-338. [PubMed: 9512423]

20. Simpson F, Whitehead JP. Adiponectin-it's all about the modifications. Int J Biochem Cell Biol. 2010; 42(6):785-788. [PubMed: 20044026]

21. Tsao TS, Tomas E, Murrey HE, et al. Role of disulfide bonds in Acrp30/adiponectin structure and signaling specificity. Different oligomers activate different signal transduction pathways. J Biol Chem. 2003; 278(50):50810-50817. [PubMed: 14522956]

22. Hug C, Wang J, Ahmad NS, et al. T-cadherin is a receptor for hexameric and high-molecularweight forms of Acrp30/adiponectin. Proc Natl Acad Sci U S A. 2004; 101(28):10308-10313. [PubMed: 15210937]

23. Pajvani UB, Du X, Combs TP, et al. Structure-function studies of the adipocyte-secreted hormone Acrp30/adiponectin. Implications fpr metabolic regulation and bioactivity. J Biol Chem. 2003; 278(11):9073-9085. [PubMed: 12496257]

24. Lara-Castro C, Luo N, Wallace P, et al. Adiponectin multimeric complexes and the metabolic syndrome trait cluster. Diabetes. 2006; 55(1):249-259. [PubMed: 16380500]

25. Vasseur F, Meyre D, Froguel P. Adiponectin, type 2 diabetes and the metabolic syndrome: lessons from human genetic studies. Expert Rev Mol Med. 2006; 8(27):1-12. [PubMed: 17112391]

26. Halberg N, Schraw TD, Wang ZV, et al. Systemic fate of the adipocyte-derived factor adiponectin. Diabetes. 2009; 58(9):1961-1970. [PubMed: 19581422]

27. Richards AA, Colgrave ML, Zhang J, et al. Sialic acid modification of adiponectin is not required for multimerization or secretion but determines half-life in circulation. Mol Endocrinol. 2010; 24(1):229-239. [PubMed: 19855092]

28. Schraw T, Wang ZV, Halberg N, et al. Plasma adiponectin complexes have distinct biochemical characteristics. Endocrinology. 2008; 149(5):2270-2282. [PubMed: 18202126]

29. Pajvani UB, Hawkins M, Combs TP, et al. Complex distribution, not absolute amount of adiponectin, correlates with thiazolidinedione-mediated improvement in insulin sensitivity. J Biol Chem. 2004; 279(13):12152-12162. [PubMed: 14699128]

30. Fruebis J, Tsao TS, Javorschi S, et al. Proteolytic cleavage product of 30-kDa adipocyte complement-related protein increases fatty acid oxidation in muscle and causes weight loss in mice. Proc Natl Acad Sci U S A. 2001; 98(4):2005-2010. [PubMed: 11172066]

31. Waki H, Yamauchi T, Kamon J, et al. Generation of globular fragment of adiponectin by leukocyte elastase secreted by monocytic cell line THP-1. Endocrinology. 2005; 146(2):790-796. [PubMed: 15528304]

32. Yamauchi T, Kamon J, Ito Y, et al. Cloning of adiponectin receptors that mediate antidiabetic metabolic effects. Nature. 2003; 423(6941):762-769. [PubMed: 12802337] 
33. Ranscht B, Dours-Zimmermann MT. T-cadherin, a novel cadherin cell adhesion molecule in the nervous system lacks the conserved cytoplasmic region. Neuron. 1991; 7(3):391-402. [PubMed: 1654948]

34. Vestal DJ, Ranscht B. Glycosyl phosphatidylinositol--anchored T-cadherin mediates calciumdependent, homophilic cell adhesion. J Cell Biol. 1992; 119(2):451-461. [PubMed: 1400585]

35. Ranscht B. Bradshaw R, Dennis E. Cadherins: Interactions and Regulation of Adhesivity. Handbook in Cell Signaling. 2003:889-899.

36. Yamauchi T, Kadowaki T. Adiponectin receptor as a key player in healthy longevity and obesityrelated diseases. Cell Metab. 2013; 17(2):185-196. [PubMed: 23352188]

37. Holland WL, Miller RA, Wang ZV, et al. Receptor-mediated activation of ceramidase activity initiates the pleiotropic actions of adiponectin. Nat Med. 2011; 17(1):55-63. [PubMed: 21186369]

38. Hebbard LW, Garlatti M, Young LJ, et al. T-cadherin supports angiogenesis and adiponectin association with the vasculature in a mouse mammary tumor model. Cancer Res. 2008; 68(5): 1407-1416. [PubMed: 18316604]

39. Parker-Duffen JL, Nakamura K, Silver M, et al. T-cadherin Is Essential for Adiponectinmediated Revascularization. J Biol Chem. 2013; 288(34):24886-24897. [PubMed: 23824191]

40. Denzel MS, Scimia MC, Zumstein PM, et al. T-cadherin is critical for adiponectin-mediated cardioprotection in mice. J Clin Invest. 2010; 120(12):4342-4352. [PubMed: 21041950]

41. Tyrberg B, Miles P, Azizian KT, et al. T-cadherin (Cdh13) in association with pancreatic betacell granules contributes to second phase insulin secretion. Islets. 2011; 3(6):327-337. [PubMed: 21975561]

42. Wu Y, Li Y, Lange EM, et al. Genome-wide association study for adiponectin levels in Filipino women identifies CDH13 and a novel uncommon haplotype at KNG1-ADIPOQ. Hum Mol Genet. 2010; 19(24):4955-4964. [PubMed: 20876611]

43. Morisaki H, Yamanaka I, Iwai N, et al. CDH13 gene coding T-cadherin influences variations in plasma adiponectin levels in the Japanese population. Hum Mutat. 2012; 33(2):402-410. [PubMed: 22065538]

44. Hanahan D, Weinberg RA. Hallmarks of cancer: the next generation. Cell. 2011; 144(5):646-674. [PubMed: 21376230]

45. Lunt SY, Vander Heiden MG. Aerobic glycolysis: meeting the metabolic requirements of cell proliferation. Annu Rev Cell Dev Biol. 2011; 27:441-464. [PubMed: 21985671]

46. Otake S, Takeda H, Suzuki Y, et al. Association of visceral fat accumulation and plasma adiponectin with colorectal adenoma: evidence for participation of insulin resistance. Clin Cancer Res. 2005; 11(10):3642-3646. [PubMed: 15897559]

47. Ishikawa M, Kitayama J, Kazama S, et al. Plasma adiponectin and gastric cancer. Clin Cancer Res. 2005; 11(2 Pt 1):466-472. [PubMed: 15701829]

48. Petridou ET, Mitsiades N, Gialamas S, et al. Circulating adiponectin levels and expression of adiponectin receptors in relation to lung cancer: two case-control studies. Oncology. 2007; 73(3-4):261-269. [PubMed: 18424891]

49. Kotani K, Wakai K, Shibata A, et al. Serum adiponectin multimer complexes and liver cancer risk in a large cohort study in Japan. Asian Pac J Cancer Prev. 2009; 10:87-90. [PubMed: 20553088]

50. Arano T, Nakagawa H, Tateishi R, et al. Serum level of adiponectin and the risk of liver cancer development in chronic hepatitis C patients. Int J Cancer. 2011; 129(9):2226-2235. [PubMed: 21170963]

51. Dalamaga M, Migdalis I, Fargnoli JL, et al. Pancreatic cancer expresses adiponectin receptors and is associated with hypoleptinemia and hyperadiponectinemia: a case-control study. Cancer Causes Control. 2009; 20(5):625-633. [PubMed: 19051043]

52. Izadi V, Farabad E, Azadbakht L. Serum adiponectin level and different kinds of cancer: a review of recent evidence. ISRN Oncol. 2012; 2012982769.

53. Fantuzzi G. Adiponectin and inflammation: consensus and controversy. J Allergy Clin Immunol. 2008; 121(2):326-330. [PubMed: 18061654]

54. Mistry T, Digby JE, Chen J, et al. The regulation of adiponectin receptors in human prostate cancer cell lines. Biochem Biophys Res Commun. 2006; 348(3):832-838. [PubMed: 16899222] 
55. Takahata C, Miyoshi Y, Irahara N, et al. Demonstration of adiponectin receptors 1 and 2 mRNA expression in human breast cancer cells. Cancer Lett. 2007; 250(2):229-236. [PubMed: 17123704]

56. Andreeva AV, Kutuzov MA. Cadherin 13 in cancer. Genes Chromosomes Cancer. 2010; 49(9): 775-790. [PubMed: 20607704]

57. Arcidiacono B, Iiritano S, Nocera A, et al. Insulin resistance and cancer risk: an overview of the pathogenetic mechanisms. Exp Diabetes Res. 2012; 2012789174.

58. Yamaji T, Iwasaki M, Sasazuki S, et al. Interaction between adiponectin and leptin influences the risk of colorectal adenoma. Cancer Res. 2010; 70(13):5430-5437. [PubMed: 20516125]

59. Steinberg GR, Kemp BE. AMPK in Health and Disease. Physiol Rev. 2009; 89(3):1025-1078. [PubMed: 19584320]

60. Kahn BB, Alquier T, Carling D, et al. AMP-activated protein kinase: ancient energy gauge provides clues to modern understanding of metabolism. Cell Metab. 2005; 1(1):15-25. [PubMed: 16054041]

61. Deepa SS, Dong LQ. APPL1: role in adiponectin signaling and beyond. Am J Physiol Endocrinol Metab. 2009; 296(1):E22-E36. [PubMed: 18854421]

62. Mao X, Kikani CK, Riojas RA, et al. APPL1 binds to adiponectin receptors and mediates adiponectin signalling and function. Nat Cell Biol. 2006; 8(5):516-523. [PubMed: 16622416]

63. Hezel AF, Bardeesy N. LKB1; linking cell structure and tumor suppression. Oncogene. 2008; 27(55):6908-6919. [PubMed: 19029933]

64. Shackelford DB, Shaw RJ. The LKB1-AMPK pathway: metabolism and growth control in tumour suppression. Nat Rev Cancer. 2009; 9(8):563-575. [PubMed: 19629071]

65. Yamauchi T, Kamon J, Waki H, et al. The fat-derived hormone adiponectin reverses insulin resistance associated with both lipoatrophy and obesity. Nat Med. 2001; 7(8):941-946. [PubMed: 11479627]

66. Luo Z, Zang M, Guo W. AMPK as a metabolic tumor suppressor: control of metabolism and cell growth. Future Oncol. 2010; 6(3):457-470. [PubMed: 20222801]

67. Beckers A, Organe S, Timmermans L, et al. Chemical inhibition of acetyl-CoA carboxylase induces growth arrest and cytotoxicity selectively in cancer cells. Cancer Res. 2007; 67(17):8180 8187. [PubMed: 17804731]

68. Orita H, Coulter J, Lemmon C, et al. Selective inhibition of fatty acid synthase for lung cancer treatment. Clin Cancer Res. 2007; 13(23):7139-7145. [PubMed: 18056164]

69. Zhan Y, Ginanni N, Tota MR, et al. Control of cell growth and survival by enzymes of the fatty acid synthesis pathway in HCT-116 colon cancer cells. Clin Cancer Res. 2008; 14(18):5735-5742. [PubMed: 18794082]

70. Chajes V, Cambot M, Moreau K, et al. Acetyl-CoA carboxylase alpha is essential to breast cancer cell survival. Cancer Res. 2006; 66(10):5287-5294. [PubMed: 16707454]

71. Brusselmans K, De Schrijver E, Verhoeven G, et al. RNA interference-mediated silencing of the acetyl-CoA-carboxylase-alpha gene induces growth inhibition and apoptosis of prostate cancer cells. Cancer Res. 2005; 65(15):6719-6725. [PubMed: 16061653]

72. Adekola K, Rosen ST, Shanmugam M. Glucose transporters in cancer metabolism. Curr Opin Oncol. 2012; 24(6):650-654. [PubMed: 22913968]

73. Pollak M. Insulin and insulin-like growth factor signalling in neoplasia. Nat Rev Cancer. 2008; 8(12):915-928. [PubMed: 19029956]

74. Fresno Vara JA, Casado E, de Castro J, et al. PI3K/Akt signalling pathway and cancer. Cancer Treat Rev. 2004; 30(2):193-204. [PubMed: 15023437]

75. Guertin DA, Sabatini DM. Defining the role of mTOR in cancer. Cancer Cell. 2007; 12(1):9-22. [PubMed: 17613433]

76. Sengupta S, Peterson TR, Sabatini DM. Regulation of the mTOR complex 1 pathway by nutrients, growth factors, and stress. Mol Cell. 2010; 40(2):310-322. [PubMed: 20965424]

77. Shaw RJ, Cantley LC. Ras, PI(3)K and mTOR signalling controls tumour cell growth. Nature. 2006; 441(7092):424-430. [PubMed: 16724053]

78. Orlova KA, Crino PB. The tuberous sclerosis complex. Ann N Y Acad Sci. 2010; 1184:87-105. [PubMed: 20146692] 
79. Inoki K, Li Y, Xu T, et al. Rheb GTPase is a direct target of TSC2 GAP activity and regulates mTOR signaling. Genes Dev. 2003; 17(15):1829-1834. [PubMed: 12869586]

80. Jones RG, Plas DR, Kubek S, et al. AMP-activated protein kinase induces a p53-dependent metabolic checkpoint. Mol Cell. 2005; 18(3):283-293. [PubMed: 15866171]

81. Moon HS, Liu X, Nagel JM, et al. Salutary effects of adiponectin on colon cancer: in vivo and in vitro studies in mice. Gut. 2013; 62(4):561-570. [PubMed: 22735569]

82. Kim AY, Lee YS, Kim KH, et al. Adiponectin Represses Colon Cancer Cell Proliferation via AdipoR1- and -R2-Mediated AMPK Activation. Molecular Endocrinology. 2010; 24(7):14411452. [PubMed: 20444885]

83. Polakis P. Wnt signaling in cancer. Cold Spring Harb Perspect Biol. 2012; 4(5)

84. Liu C, Li Y, Semenov M, et al. Control of beta-catenin phosphorylation/degradation by a dualkinase mechanism. Cell. 2002; 108(6):837-847. [PubMed: 11955436]

85. Wang Y, Lam JB, Lam KS, et al. Adiponectin modulates the glycogen synthase kinase- 3beta/betacatenin signaling pathway and attenuates mammary tumorigenesis of MDA-MB-231 cells in nude mice. Cancer Res. 2006; 66(23):11462-11470. [PubMed: 17145894]

86. Liu J, Lam JB, Chow KH, et al. Adiponectin stimulates Wnt inhibitory factor-1 expression through epigenetic regulations involving the transcription factor specificity protein 1 . Carcinogenesis. 2008; 29(11):2195-2202. [PubMed: 18701434]

87. Garofalo C, Koda M, Cascio S, et al. Increased expression of leptin and the leptin receptor as a marker of breast cancer progression: possible role of obesity-related stimuli. Clin Cancer Res. 2006; 12(5):1447-1453. [PubMed: 16533767]

88. Howard JM, Pidgeon GP, Reynolds JV. Leptin and gastro-intestinal malignancies. Obes Rev. 2010; 11(12):863-874. [PubMed: 20149119]

89. Sharma D, Wang J, Fu PP, et al. Adiponectin antagonizes the oncogenic actions of leptin in hepatocellular carcinogenesis. Hepatology. 2010; 52(5):1713-1722. [PubMed: 20941777]

90. Miyazaki T, Bub JD, Uzuki M, et al. Adiponectin activates c-Jun NH2-terminal kinase and inhibits signal transducer and activator of transcription 3. Biochem Biophys Res Commun. 2005; 333(1): 79-87. [PubMed: 15936715]

91. Yan D, Avtanski D, Saxena NK, et al. Leptin-induced epithelial-mesenchymal transition in breast cancer cells requires beta-catenin activation via Akt/GSK3- and MTA1/Wnt1 proteindependent pathways. J Biol Chem. 2012; 287(11):8598-8612. [PubMed: 22270359]

92. Maceyka M, Harikumar KB, Milstien S, et al. Sphingosine-1-phosphate signaling and its role in disease. Trends Cell Biol. 2012; 22(1):50-60. [PubMed: 22001186]

93. Pyne NJ, Pyne S. Sphingosine 1-phosphate and cancer. Nat Rev Cancer. 2010; 10(7):489-503. [PubMed: 20555359]

94. Denzel MS, Hebbard LW, Shostak G, et al. Adiponectin deficiency limits tumor vascularization in the MMTV-PyV-mT mouse model of mammary cancer. Clin Cancer Res. 2009; 15(10):32563264. [PubMed: 19447866]

95. Landskroner-Eiger S, Qian B, Muise ES, et al. Proangiogenic contribution of adiponectin toward mammary tumor growth in vivo. Clin Cancer Res. 2009; 15(10):3265-3276. [PubMed: 19447867]

96. Bergers G, Hanahan D. Modes of resistance to anti-angiogenic therapy. Nat Rev Cancer. 2008; 8(8):592-603. [PubMed: 18650835]

97. Shibata R, Ouchi N, Kihara S, et al. Adiponectin stimulates angiogenesis in response to tissue ischemia through stimulation of amp-activated protein kinase signaling. J Biol Chem. 2004; 279(27):28670-28674. [PubMed: 15123726]

98. Ouchi N, Kobayashi H, Kihara S, et al. Adiponectin stimulates angiogenesis by promoting crosstalk between AMP-activated protein kinase and Akt signaling in endothelial cells. J Biol Chem. 2004; 279(2):1304-1309. [PubMed: 14557259]

99. Lee MH, Klein RL, El-Shewy HM, et al. The adiponectin receptors AdipoR1 and AdipoR2 activate ERK1/2 through a Src/Ras-dependent pathway and stimulate cell growth. Biochemistry. 2008; 47(44):11682-11692. [PubMed: 18842004]

100. Li FY, Cheng KK, Lam KS, et al. Cross-talk between adipose tissue and vasculature: role of adiponectin. Acta Physiol (Oxf). 2011; 203(1):167-180. [PubMed: 21062420] 
101. Hadad SM, Fleming S, Thompson AM. Targeting AMPK: a new therapeutic opportunity in breast cancer. Crit Rev Oncol Hematol. 2008; 67(1):1-7. [PubMed: 18343152]

102. Guo D, Hildebrandt IJ, Prins RM, et al. The AMPK agonist AICAR inhibits the growth of EGFRvIII-expressing glioblastomas by inhibiting lipogenesis. Proc Natl Acad Sci U S A. 2009; 106(31):12932-12937. [PubMed: 19625624]

103. Otvos L Jr, Haspinger E, La Russa F, et al. Design and development of a peptide-based adiponectin receptor agonist for cancer treatment. BMC Biotechnol. 2011; 11:90. [PubMed: 21974986] 


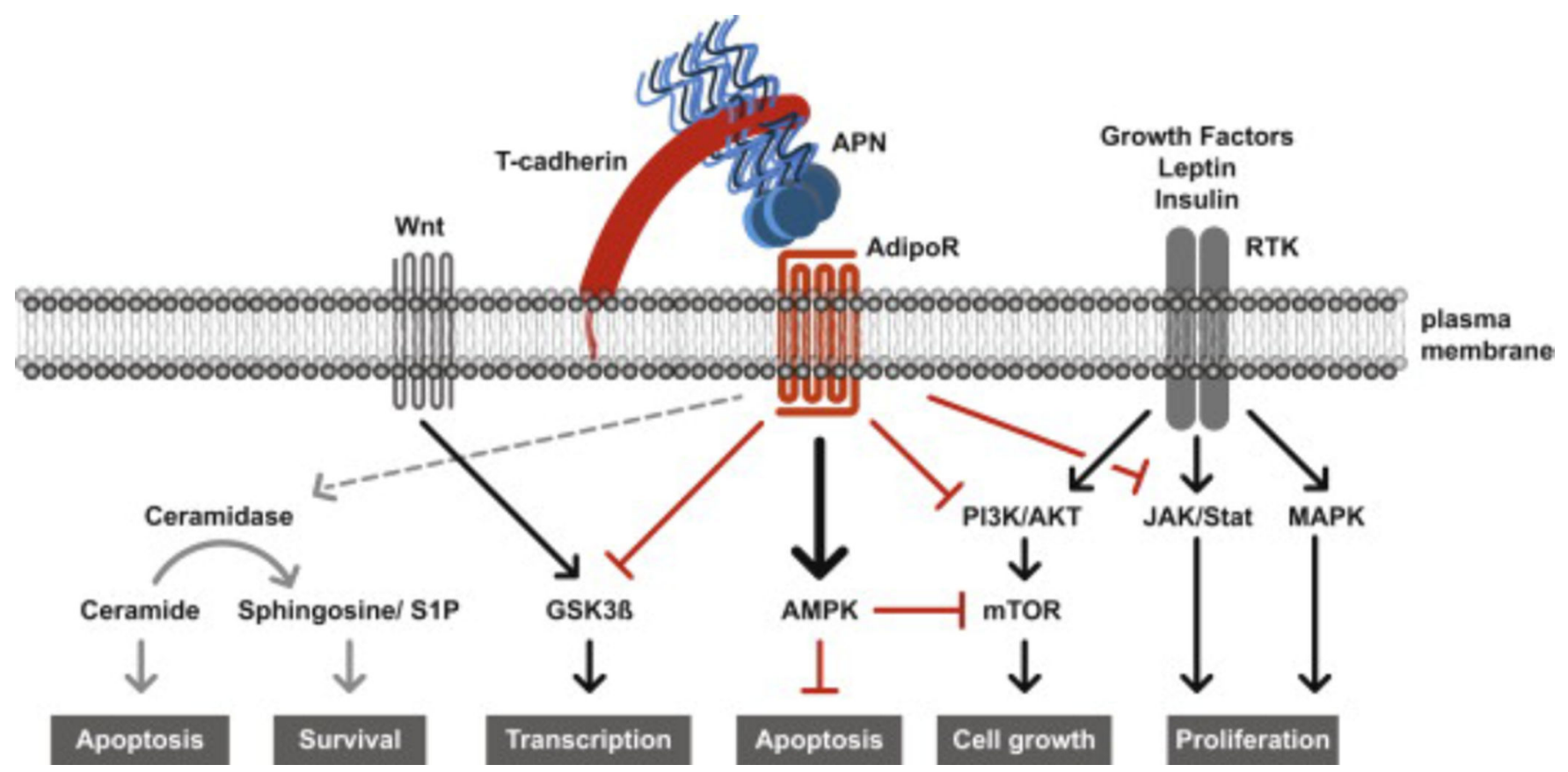

Fig. 1.

Model for APN-mediated suppression of cancer promoting pathways. In normal epithelial cells, APN is bound by T-cadherin and presented directly or indirectly to AdipoR1/R2 to inhibit signaling pathways activated in neoplasia. APN-activates AMPK, and inhibits PI3K/AKT, mTOR, MAPK and JAK/Stat pathways, or directly affects GSK3 $\beta$ to suppress cancer promoting pathways. Cancer cells down-regulate T-cadherin while AdipoR1/R2 expression persists and cancer promoting pathways prevail. One model is that ceramidase activity associated with AdipoR1/R2 weighs the balance in favor of cancer cell survival. T-cadherin expressed in the tumor vasculature promotes cancer as a pro-angiogenic factor in cooperation with APN (not shown $[38,39])$. Black arrows, activating pathways; red lines with bar: inhibitory pathways. 